\title{
Investigation of a synthetic diamond detector response in kilovoltage photon beams
}

Vaiva Kaveckyte, Linda Persson, Alexandr Malusek, Hamza Benmakhlouf, Gudrun Alm Carlsson and Åsa Carlsson Tedgren

The self-archived postprint version of this journal article is available at Linköping University Institutional Repository (DiVA):

http://urn.kb.se/resolve?urn=urn:nbn:se:liu:diva-163628

N.B.: When citing this work, cite the original publication.

Kaveckyte, V., Persson, L., Malusek, A., Benmakhlouf, H., Alm Carlsson, G., Carlsson Tedgren, Å., (2020), Investigation of a synthetic diamond detector response in kilovoltage photon beams, Medical physics (Lancaster). https://doi.org/10.1002/mp.13988

Original publication available at:

https://doi.org/10.1002/mp.13988

Copyright: Wiley

http://eu.wiley.com/WileyCDA/ 


\title{
Investigation of a synthetic diamond detector response in kilovoltage photon beams
}

\author{
Vaiva Kaveckyte, ${ }^{\mathrm{a}, \mathrm{b})}$, Linda Persson ${ }^{\mathrm{c}}$, Alexandr Malusek $^{\mathrm{a})}$, Hamza Benmakhlouf ${ }^{\mathrm{b})}$, Gudrun Alm \\ Carlsson $^{\text {a) }}$ and Åsa Carlsson Tedgren ${ }^{\mathrm{a}, \mathrm{b})}$

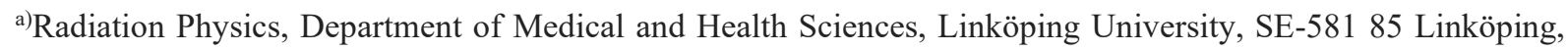 \\ Sweden \\ ${ }^{b}$ Department of Medical Radiation Physics and Nuclear Medicine, Karolinska University Hospital, SE-171 76 \\ Stockholm, Sweden

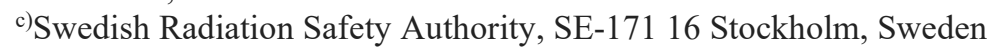 \\ E-mail of the corresponding author: vaiva.kaveckyte@liu.se
}

\begin{abstract}
Purpose: An important characteristic of radiation dosimetry detectors is their energy response that consists of absorbed-dose and intrinsic energy responses. The former can be characterized using Monte Carlo (MC) simulations, whereas the latter (i.e., detector signal per absorbed dose to detector) is extracted from experimental data. Such a characterization is especially relevant when detectors are used in non-relative measurements at a beam quality that differs from the calibration beam quality. Having in mind possible application of synthetic diamond detectors (microDiamond PTW 60019, Freiburg, Germany) for non-relative dosimetry of low-energy brachytherapy (BT) beams, we determined their intrinsic and absorbed-dose energy responses in $25-250 \mathrm{kV}$ beams relative to a ${ }^{60} \mathrm{Co}$ beam, which is usually the reference beam quality for detector calibration in radiotherapy.
\end{abstract}

Material and Methods: Three microDiamond detectors and, for comparison, two silicon diodes (PTW 60017) were calibrated in terms of air-kerma free in air in six x-ray beam qualities (from 25 to $250 \mathrm{kV}$ ) and in terms of absorbed dose to water in a ${ }^{60} \mathrm{Co}$ beam at the national metrology laboratory in Sweden. The PENELOPE/penEasy MC radiation transport code was used to calculate absorbed-dose energy response of detectors (modeled based on blueprints) relative to air and water depending on calibration conditions. The MC results were used to 
extract the relative intrinsic energy response of detectors from the overall energy response. Measurements using an independent setup with a single ophthalmic BEBIG I25.S16 ${ }^{125}$ I BT seed (effective photon energy of $28 \mathrm{keV}$ ) were used as a qualitative check of the extracted intrinsic energy response correction factors. Additionally, the impact of the thickness of the active volume as well as the presence of extra-cameral components on the absorbed-dose energy response of a microDiamond detector was studied using MC.

Results: The relative intrinsic energy response of microDiamond detectors was higher by a factor of two in 25 and $50 \mathrm{kV}$ beams compared to the ${ }^{60} \mathrm{Co}$ beam. The variation in the relative intrinsic energy response of silicon diodes was within $10 \%$ over the investigated photon energy range. The use of relative intrinsic energy response correction factors improved the agreement among the absorbed dose to water values determined using microDiamond detectors and silicon diodes, as well as with the TG-43 formalism-based calculations for the ${ }^{125}$ I seed. MC study of detector design features provided a possible explanation for intra-detector response variation at low-energy photon beams by differences in the effective thickness of the active volume.

Conclusion: MicroDiamond detectors had a non-negligible variation in the relative intrinsic energy response (factor of two) which was comparable to that in the absorbed-dose energy response relative to water at low-energy photon beams. Silicon diodes, on the other hand, had an absorbed-dose energy dependence on photon energy that varied by a factor of six, whereas the intrinsic energy dependence on beam quality was within $10 \%$. It is important to decouple these two responses for a full characterization of detector energy response especially when the user and reference beam qualities differ significantly, and $\mathrm{MC}$ alone is not enough. 


\section{INTRODUCTION}

Commercial natural diamond detectors and custom-made synthetic diamond detectors have been used for dosimetry in high energy photon and electron beams and brachytherapy (BT) due to their water-equivalence (carbon atomic number $Z=6$ compared with water $Z_{\text {eff }}=7.4$ ). However, their use diminished over the years due to scarcity of high-grade natural crystals and expensive and non-reproducible manufacture of synthetic diamond crystals. The main reason was that the quality of the crystal (e.g., the amount of impurities) affected their dosimetric properties, such as dose-rate dependence and dose linearity. Recently diamond detectors have regained interest because the current manufacturing technology allows for reproducible highpurity crystal growth. Commercially-available synthetic diamond detectors microDiamond (PTW 60019, Freiburg, Germany) have been used in small-field dosimetry of high-energy photon and electron beams, ${ }^{1-4}$ as well as in proton and carbon beams. ${ }^{5-8}$ These studies showed that the detectors have low absorbed-dose energy dependence, negligible dose-rate dependence and do not require high pre-irradiation doses (up to 2 Gy). These characteristics shown in high energy beams combined with the closeness of the effective atomic numbers of water and carbon prompted a question about diamond detector's applicability in low-energy photon beams, such as BT and diagnostic X-ray fields. It has been shown that the microDiamond detector is suitable for determination of absorbed dose to water ${ }^{9}$ and for relative measurements in HDR ${ }^{192} \mathrm{Ir}$ beams, ${ }^{10}$ and percentage depth-dose curve determination in kilovoltage $(\mathrm{kV}) \mathrm{x}$-ray beams. ${ }^{11}$

Having in mind the possible application of microDiamond detectors for experimental nonrelative dosimetry of, e.g., electronic BT treatments (40 to $100 \mathrm{kV})$ or treatments using ${ }^{125} \mathrm{I}$ seeds, the purpose of this study was to further investigate the response of microDiamond detectors at low photon energies (from 25 to $250 \mathrm{kV} \mathrm{x}$-ray beams) and compare it with that in ${ }^{a}{ }^{60} \mathrm{Co}$ beam. Determination of absorbed dose to water around BT sources in absolute units of Gy is required to verify Monte Carlo (MC) single-source dosimetry data before use in treatment 
planning. ${ }^{12,13}$ The currently-recommended dosimetry system is lithium fluoride thermoluminescent dosimeters (LiF TLD) calibrated in ${ }^{60} \mathrm{Co}$ or $6 \mathrm{MV}$ beams using external beam protocols. ${ }^{14,15}$ Although these detectors have low absorbed-dose energy dependence, they have an intrinsic energy dependence at low photon energies compared to a high-energy photon calibration beam. ${ }^{16-18}$ Additionally, they are passive readout detectors which are less convenient to use compared to direct readout detectors.

To investigate whether microDiamond detectors have an intrinsic energy dependence, we extracted the intrinsic energy response (detector signal per average absorbed dose to detector) from the overall energy response. We accounted for the absorbed-dose energy response of detectors using blueprints and detailed MC simulations and, given the complexity of detector construction, further investigated intra-detector variation among the three tested microDiamond detectors. For comparison, we included two silicon diode detectors (PTW 60017, Freiburg, Germany) that have the same outer dimensions and fit into our measurement setup. Furthermore, it was of interest to compare the intrinsic energy response of two solid-state direct readout detectors.

The obtained results may allow for better understanding of detector response and could be used to optimize future design so that the detector's energy dependence and radiation field perturbations in low energy photon beams such as those used in BT are minimized.

\section{MATERIALS AND METHODS}

\subsection{Dosimetry formalism}

The quantity of interest in radiation therapy dosimetry usually is absorbed dose to water, hence detectors used for non-relative measurements are calibrated in terms of absorbed dose to water in a reference beam quality $Q_{0}$. Although it is desirable that calibration beam quality would resemble user's beam quality $Q$, it is not always possible and detector response to beam quality 
$Q$ has to be corrected for possible differences between the two beam qualities. Absorbed dose to water in the beam quality $Q$ can be determined as follows

$$
D_{\mathrm{w}, Q}=M_{Q} N_{D, \mathrm{w}, Q_{0}} k_{Q, Q_{0}},
$$

where $M_{Q}$ is the reading of the dosimeter corrected for influence quantities in a measurement phantom in beam quality $Q, N_{D, \mathrm{w}, Q_{0}}$ is the calibration coefficient of the detector in terms of absorbed dose to water in the reference beam quality $Q_{0}$, and $k_{Q, Q_{0}}$ is the beam quality correction factor. $N_{D, \mathrm{w}, Q_{0}}$ is determined as $\left[D_{\mathrm{w}} / M\right]_{Q_{0}}$, which relates the detector signal $M$ to the absorbed dose to water $D_{\mathrm{w}}$ and defines the overall response of detector in beam quality $Q_{0}$. The beam quality correction factor $k_{Q, Q_{0}}$ can be calculated as follows

$$
k_{Q, Q_{0}}=\frac{\left[D_{\mathrm{w}} / \bar{D}_{\mathrm{det}}\right]_{Q}}{\left[D_{\mathrm{w}} / \bar{D}_{\mathrm{det}}\right]_{Q_{0}}} \cdot \frac{\left[M / \bar{D}_{\mathrm{det}}\right]_{Q_{0}}}{\left[M / \bar{D}_{\mathrm{det}}\right]_{Q}}=\frac{\left[D_{\mathrm{w}} / \bar{D}_{\mathrm{det}}\right]_{Q}}{\left[D_{\mathrm{w}} / \bar{D}_{\mathrm{det}}\right]_{Q_{0}}} \cdot \frac{R_{Q_{0}}}{R_{Q}},
$$

where $\bar{D}_{\text {det }}$ is the average absorbed dose to the active volume of the detector at the point of measurement, and $D_{\mathrm{w}}$ is the corresponding absorbed dose to a small volume of water at the point of measurement in the absence of the detector in beam qualities $Q$ and $Q_{0}$. Quantities $R_{Q}$ and $R_{Q_{0}}$ characterize the physical signal generation and its collection-efficiency in the detector at a given beam quality, i.e., detector signal per average absorbed dose to the detector, $R=$ $\left[M / \bar{D}_{\mathrm{det}}\right]_{i}\left(i=Q, Q_{0}\right)$. This quantity may depend on, e.g., the ionization density of secondary electrons, dose-rate or the accumulated absorbed dose in the detector. Since it cannot be obtained through $\mathrm{MC}$ calculations (unless they implement mathematical models describing the conversion from absorbed dose to detector signal), it has to be determined from measurements. It can be extracted from experimentally-determined detector calibration coefficients by accounting for absorbed-dose energy dependence between water and detector medium in a given beam quality as 


$$
R_{Q_{0}}=\left[\frac{M}{\bar{D}_{\mathrm{det}}}\right]_{Q_{0}}=\frac{1}{N_{D, \mathrm{w}, Q_{0}}} \cdot\left[\frac{D_{\mathrm{w}}}{\bar{D}_{\mathrm{det}}}\right]_{Q_{0}}=\left[\frac{M}{D_{\mathrm{w}}}\right]_{Q_{0}} \cdot\left[\frac{D_{\mathrm{w}}}{\bar{D}_{\mathrm{det}}}\right]_{Q_{0}},
$$

where $N_{D, \mathrm{w}, Q_{0}}$ is the detector calibration coefficient in terms of absorbed dose to water determined experimentally and traceable to the primary standards. Usually $\left[D_{\mathrm{w}} / \bar{D}_{\mathrm{det}}\right]_{Q_{0}}$ is an MC calculated ratio of absorbed dose to water $D_{\mathrm{w}}$ at a measurement point in the absence of the detector and the average absorbed dose $\bar{D}_{\text {det }}$ to the active volume of the detector at the point of measurement. When detectors are calibrated in terms of air-kerma free in air, the same approach applies but $N_{D, \mathrm{w}, Q_{0}}$ is replaced with $N_{K \text {,air }}$ which is the detector calibration coefficient in terms of air-kerma free in air at a given beam quality $Q$ and traceable to the primary standards. Similarly, the MC calculated quantity $D_{\mathrm{w}}$ in Eq. (1.3) is replaced with the calculated $K_{\text {air }}$ values.

Finally, the relative intrinsic energy dependence can be written as

$$
\frac{R_{Q_{0}}}{R_{Q}}=\frac{\left[M / \bar{D}_{\mathrm{det}}\right]_{Q_{0}}}{\left[M / \bar{D}_{\mathrm{det}}\right]_{Q}}=\frac{\left[M / D_{\mathrm{w}}\right]_{Q_{0}}}{\left[M / D_{\mathrm{w}}\right]_{Q}} \cdot \frac{\left[D_{\mathrm{w}} / \bar{D}_{\mathrm{det}}\right]_{Q_{0}}}{\left[D_{\mathrm{w}} / \bar{D}_{\mathrm{det}}\right]_{Q}}
$$

For a detector calibrated in terms of air-kerma free in air $K_{\text {air }}$ in beam quality $Q$, Eq. (1.4) becomes

$$
\frac{R_{Q_{0}}}{R_{Q}}=\frac{\left[M / \bar{D}_{\mathrm{det}}\right]_{Q_{0}}}{\left[M / \bar{D}_{\mathrm{det}}\right]_{Q}}=\frac{\left[M / D_{\mathrm{w}}\right]_{Q_{0}}}{\left[M / K_{\mathrm{air}}\right]_{Q}} \cdot \frac{\left[D_{\mathrm{w}} / \bar{D}_{\mathrm{det}}\right]_{Q_{0}}}{\left[K_{\mathrm{air}} / \bar{D}_{\mathrm{det}}\right]_{Q}}
$$

It is important to note that $N_{D, \mathrm{w}, Q_{0}}$, and subsequently $k_{Q, Q_{0}}$ (given by Eq. (1.2)), consists of two parts. The absorbed-dose energy-response of a detector, i.e., the ratios $\left[D_{\mathrm{w}} / \bar{D}_{\mathrm{det}}\right]_{Q}$ and $\left[D_{\mathrm{w}} / \bar{D}_{\mathrm{det}}\right]_{Q_{0}}$, describes the energy absorption properties of a detector compared to those of the medium of interest. This absorbed-dose energy response of a detector can be quantified using MC simulations for a given beam quality when detector geometry and materials are well known. The ratio $R_{Q_{0}} / R_{Q}$ accounts for the remaining properties. 
When detectors are calibrated in a ${ }^{60} \mathrm{Co}$ or MV beam, $k_{Q, Q_{0}}$ is commonly calculated using MC simulations which neglect the energy dependence of the intrinsic energy response of the detector $R(E)$. In practice, this energy dependence is not accounted for in high-energy photon beam dosimetry using ion chambers because the variation in energy needed to create an ion pair is assumed to be negligible. ${ }^{15}$ However, it cannot be ignored for other detector types when they are calibrated in high-energy beams but used in low energy beams, e.g., in BT. It has been shown that, for instance, some types of TLDs, ${ }^{16-18}$ radio-photoluminescent glass dosimeters ${ }^{19}$ and EPR dosimeters ${ }^{20,21}$ calibrated in high energy photon beams exhibit a non-negligible variation in their intrinsic energy response when used in $\mathrm{kV}$ beams. This was attributed mainly to the difference in the ionization density of secondary electrons between the calibration beam and the measurement beams (where the effective photon energy ranges from 13 to $375 \mathrm{keV}$ ) that affected either radical production in EPR dosimeters or traps formation in TLDs in addition to the dependence on TLD readout process. ${ }^{18}$

\subsection{Detectors and calibration}

Three microDiamond detectors PTW 60019 [referred to as mD1 (s/n 122518), mD2 (s/n 122840) and mD3 (s/n 122043)] and two PTW 60017 silicon diodes [referred to as SiD1 (s/n 780) and $\mathrm{SiD} 2(\mathrm{~s} / \mathrm{n}$ 974)] were used. The nominal active volume of microDiamond was a $1 \mu \mathrm{m}$ thickness cylinder with $1.1 \mathrm{~mm}$ radius. The silicon diode had a $30 \mu \mathrm{m}$ thickness cylindrical active volume with a radius of $0.6 \mathrm{~mm}$. All detectors were calibrated in terms of absorbed dose to water in a ${ }^{60} \mathrm{Co}$ beam following the TRS-398 protoco $^{15}$ and in terms of air-kerma free in air in six CCRI x-ray beam qualities ${ }^{22}$ from 25 to $250 \mathrm{kV}$ (see Table 1) at the Swedish Radiation Safety Authority Secondary Standards Dosimetry Laboratory (SSDL). At calibration in the 25 and $50 \mathrm{kV}$ beams, the reference-point (marked with a red or grey ring) of the detector was placed at $50 \mathrm{~cm}$ from the source where the diameter of the irradiation field was $9.6 \mathrm{~cm}$. At other $\mathrm{kV}$ beam qualities, the respective values of distance to the source and irradiation field diameter 
were $100 \mathrm{~cm}$ and $10.5 \mathrm{~cm}$. The geometric depth of the active area was at $0.95 \mathrm{~mm}$ and $0.76 \mathrm{~mm}$ measured from the detector tip for microDiamond and silicon diode detectors respectively. Detectors were oriented with their longitudinal axis parallel to the beam direction. MicroDiamond detectors were pre-irradiated with approximately 5 Gy before measurements as recommended in the specifications.

Table 1 X-ray beams used for detector calibration in terms of air-kerma free in air. Effective energy was calculated as the energy of a monoenergetic beam which would have the same half-value layer as a given spectrum. Airkerma rate is given at a source-to-detector distance where detectors were calibrated. Beam quality data were provided by the SSDL.

\begin{tabular}{lcccc}
\hline \hline \multicolumn{1}{c}{ Beam } & $\begin{array}{c}\text { Generating } \\
\text { potential }(\mathrm{kV})\end{array}$ & $\begin{array}{c}\text { Effective } \\
\text { energy }(\mathrm{keV})\end{array}$ & $\begin{array}{c}\text { HVL } \\
(\mathrm{mm})\end{array}$ & $\begin{array}{c}\text { Air-kerma rate } \\
(\mathrm{mGy} / \mathrm{s})\end{array}$ \\
\hline \hline CCRI-25 & 25 & 13.9 & $0.25 \mathrm{Al}$ & 2.1 \\
CCRI-50(b) & 50 & 22.6 & $1.00 \mathrm{Al}$ & 3.0 \\
CCRI-100 & 100 & 38.5 & $0.14 \mathrm{Cu}$ & 1.0 \\
CCRI-135 & 135 & 59.4 & $0.47 \mathrm{Cu}$ & 0.9 \\
CCRI-180 & 180 & 77.7 & $0.93 \mathrm{Cu}$ & 1.3 \\
CCRI-250 & 250 & 126.9 & $2.49 \mathrm{Cu}$ & 1.6 \\
\hline \hline
\end{tabular}

\subsection{Monte Carlo simulations}

MC simulations were used i) to calculate the absorbed-dose energy dependence $\left(D_{\mathrm{w}} / \bar{D}_{\mathrm{det}}\right.$ and $\left.K_{\text {air }} / \bar{D}_{\text {det }}\right)$ of each detector type in a given beam quality so that the intrinsic energy response could be calculated using Eqs. (1.4) and (1.5), and ii) to investigate the impact of detector design features, such as the thickness of the active volume and the presence of extra-cameral components, on its absorbed-dose energy response.

Calculations were made using a general-purpose main program penEasy ${ }^{23}$ (v. 2015-05-30) for the PENELOPE (v. 2014) MC system. ${ }^{24}$ PENELOPE-2014 implements detailed simulation of photon and electron transport. Photon interactions include photoelectric effect, Compton scattering based on impulse approximation ${ }^{25}$ accounting for Doppler broadening and electron binding effects and Rayleigh scattering using the non-relativistic atomic form factors from the 
EPDL tables. ${ }^{26}$ Emission of characteristic x-rays and Auger electrons is not simulated in vacancies above the N shell. Though PENELOPE-2014 allows detailed simulation of electron interactions, a mixed simulation scheme was used to speed up the calculations even in the thin active volume of the detector. A built-in geometry package PENGEOM was used to define detector and experimental setup geometries with quadric surfaces. Calibration beam spectra (see Table 1) were taken from Adolfsson et al. ${ }^{20}$ The material composition and geometry of microDiamond and PTW 60017 silicon diode detectors were modeled according to detailed blueprints obtained under a non-disclosure agreement from the manufacturer.

$D_{\mathrm{w}, \mathrm{MC}}$ and $\bar{D}_{\mathrm{det}, \mathrm{MC}}$ from Eq. (1.5) were calculated by dividing the scored energy imparted to a small volume of water in the absence of the detector and to the active volume of the detector, respectively, with the mass of the volume. The scoring volume of water was equal to the nominal active volume of the microDiamond detector. $K_{\text {air,MC }}$ for a given beam quality was calculated as

$$
K_{\mathrm{air}, \mathrm{MC}}=\int_{E} E \phi_{E}\left(\frac{\mu_{\mathrm{tr}}}{\rho}(\mathrm{E})\right)_{\mathrm{air}} d E
$$

The photon fluence differential in energy $\phi_{E}$ was scored using photon-fluence track-length tally in a volume of air equal to the active volume of the microDiamond detector. Since the radiative fraction $g$ is negligible in air in the investigated $\mathrm{x}$-ray energy range below $250 \mathrm{keV}$, the mass energy-transfer coefficient $\left(\mu_{\mathrm{tr}} / \rho\right)_{\text {air }}$ was approximated by the mass energy-absorption coefficient $\left(\mu_{\mathrm{en}} / \rho\right)_{\text {air }}$. For consistency, numerical values were calculated using the mutren code included in the PENELOPE-2014 package. The difference between these and NIST values ${ }^{27}$ was estimated to be up to $3 \%$ below $100 \mathrm{keV}$ for the air medium. ${ }^{28}$

Both electron and photon transports were used to calculate $D_{\mathrm{w}, \mathrm{MC}}$ and $\bar{D}_{\mathrm{det}, \mathrm{MC}}$ for a given beam quality in Eq. (1.5). Electron cutoff energies were $1 \mathrm{keV}$ in the scoring volume and neighboring regions and $10 \mathrm{keV}$ in other regions. Photon transport cutoff energy was $1 \mathrm{keV}$ in all regions. 
The threshold kinetic energy for inelastic collisions ( $\mathrm{W}_{\mathrm{CC}}$ ) was $10 \mathrm{keV}$, the threshold energy for radiative collisions $\left(\mathrm{W}_{\mathrm{CR}}\right)$ was $1 \mathrm{keV}$, the elastic collision parameters $\left(\mathrm{C}_{1}\right.$ and $\left.\mathrm{C}_{2}\right)$ were 0.05 in the scoring volume and neighboring regions within the secondary electron range and 0.1 in other regions.

\subsection{Measurements and calculations for an ${ }^{125}$ I BT seed}

To test the MC model used for the determination of $R_{Q} / R_{Q_{0}}$ in calibration beams, an independent set of measurements was performed with a single ${ }^{125}$ I seed I25.S16-C (Eckert \& Ziegler Bebig, Germany). The seed was placed at the center of a cubic PMMA phantom (20 x $20 \times 20 \mathrm{~cm}^{3}$ ). Measurements were made at 5 and $10 \mathrm{~mm}$ distances from the detector tip to the seed center. Longitudinal axis of the detector was perpendicular to the longitudinal axis of the seed and aligned with its center. Three series of ten one-minute measurements were made with all detectors using an electrometer (PTW Unidos E, Freiburg, Germany) operating in the integrated current mode at zero nominal voltage. The average of all readings of a detector at a given distance was used in further calculations and data analysis.

The PENELOPE/penEasy code was used to score energy imparted to the nominal active volume of the detector and in a volume of water in the absence of the detector (the volume was taken as equal to that of the nominal active volume of microDiamond detector). A full seed geometry was modeled based on the I25.S06 seed model $^{29}$ (it is identical to the I25.S16 model in its design). The same electron and photon transport parameters were used as described in Section 2.32.3. The obtained values were used to calculate $D_{\mathrm{w}} / \bar{D}_{\mathrm{det}}$ to account for the absorbed-dose energy dependence of the detectors and the absorbed dose to water was determined as

$$
D_{\mathrm{w}}=M_{Q} N_{D, \mathrm{w}, Q_{0}} k_{Q, Q_{0}}=M_{Q} N_{D, w, Q_{0}} \frac{\left[D_{\mathrm{w}} / \bar{D}_{\mathrm{det}}\right]_{Q}^{\mathrm{MC}}}{\left[D_{\mathrm{w}} / \bar{D}_{\mathrm{det}}\right]_{Q_{0}}^{\mathrm{MC}}} \frac{R_{Q_{0}}}{R_{Q}},
$$

where $Q$ corresponds to the ${ }^{125} \mathrm{I}$ beam quality at either 5 or $10 \mathrm{~mm}, M_{Q}$ is the corresponding average detector reading, and $Q_{0}$ is the ${ }^{60} \mathrm{Co}$ calibration beam quality. Other quantities are 
described in Section 2.1. The $R_{Q}$ value for the photon energy of $28 \mathrm{keV}$, which is the fluenceweighted photon energy of the ${ }^{125} \mathrm{I}$ seed, was obtained by interpolating $R_{Q}$ values from $\mathrm{kV}$ beam calibration data. Furthermore, we compared our experimentally-determined values of $D_{\mathrm{w}}$ with the TG-43 formalism ${ }^{12}$ based BT treatment planning system (TPS) BrachyVision (version 13.6) (Varian Medical Systems, Inc., Palo Alto, CA, USA). Source air-kerma rate specified by the vendor was used for calculations. This value was verified using a Standard Imaging HDR 1000 Plus well-type ion chamber calibrated at SSDL and it agreed to within $2 \%$, which is within the relative expanded measurement uncertainty.

\subsection{Uncertainty analysis}

Uncertainty analysis was carried out following the "Guide to the expression of uncertainty in measurement". ${ }^{30}$ All uncertainties are given as expanded relative uncertainties $(k=2)$. Uncertainties in MC-calculated quantities account only for statistical uncertainties. Uncertainty analysis of the intrinsic energy response $R_{Q}$ is given in Table 2 .

Table 2 Uncertainty components in the intrinsic energy response $\boldsymbol{R}_{\boldsymbol{Q}}$ of detectors used in the study. All values are given with a coverage factor $k=2$.

\begin{tabular}{lccc}
\hline \multicolumn{1}{c}{ Component } & \multirow{2}{*}{ Type } & \multicolumn{2}{c}{ Uncertainty (\%) } \\
& & 25 and $50 \mathrm{kV}$ & Other kV beams \\
\hline \hline Detector calibration coefficient $N_{K, \text { air }}$ & $\mathrm{B}$ & 1.4 & 0.8 \\
MC-calculated $K_{\text {air,MC }}$ & & & \\
$\quad$ Statistical & $\mathrm{A}$ & 0.2 & 0.1 \\
$\quad$ Source spectrum & $\mathrm{B}$ & 0.6 & 0.4 \\
MC-calculated $\bar{D}_{\text {det,MC }}$ & & & 0.4 \\
$\quad$ Statistical & $\mathrm{A}$ & 0.4 & 1.0 \\
Total combined uncertainty in $R_{Q}$ & & 1.6 & \\
\hline \hline
\end{tabular}

The combined relative expanded uncertainty in $R_{Q_{0}}(1.2 \%$ for $k=2)$ was calculated as a quadratic sum of the individual uncertainties of $N_{D, \mathrm{w}, Q_{0}}$, which was $1.0 \%$, and $D_{\mathrm{w}, \mathrm{MC}}$ and $\bar{D}_{\text {det,MC }}$, both of which were $0.4 \%$. 
The impact of possible variation in manufacturer's provided atomic composition and density of detector components on MC-calculated quantities was not evaluated but possible influence is discussed in Section 4.2. Uncertainty analysis of experimentally-determined absorbed dose to water $D_{\mathrm{w}}$ in the ${ }^{125} \mathrm{I}$ beam is given in Table 3.

Table 3 Uncertainty components in the experimentally determined absorbed dose to water $\boldsymbol{D}_{\mathbf{w}}$ in the ${ }^{125} \mathrm{I}$ beam using microDiamond and silicon diode detectors. Uncertainties with a range corresponding to different source-todetector distances (SDDs) and detector types. All values are given with a coverage factor $k=2$.

\begin{tabular}{lcc}
\hline \multicolumn{1}{c}{ Component } & Type & Uncertainty (\%) \\
\hline \hline Detector readout $M_{Q}$ & & $5.2-9.2$ \\
Signal readout & $\mathrm{A}$ & $1.8-6.0$ \\
Detector positioning (5 and $10 \mathrm{~mm}$ SDD) & $\mathrm{B}$ & 8 and 4 \\
Electrometer calibration & $\mathrm{B}$ & 0.5 \\
Detector calibration coefficient $N_{D, \mathrm{w}, Q_{0}}$ & $\mathrm{~B}$ & 1.0 \\
MC-calculated $\left[D_{\mathrm{w}} / \bar{D}_{\text {det }}\right]$ & $\mathrm{A}$ & 0.6 \\
Relative intrinsic energy response $\left[R_{Q_{0}} / R_{Q}\right]$ & $\mathrm{B}$ & 2.0 \\
Total combined uncertainty in $D_{\mathrm{w}}$ & & $5.7-9.5$ \\
\hline \hline
\end{tabular}

The uncertainty in detector positioning was assumed to have a rectangular probability distribution with a half-width of $0.3 \mathrm{~mm}$ and was estimated based on TG-43 calculations of absorbed dose to water around ${ }^{125}$ I sources (a line source approximation).

The relative expanded uncertainty in $D_{\mathrm{w}, \mathrm{TPS}}$ for the TG-43 formalism based TPS calculations was $11.4 \%$ at $1 \mathrm{~cm}$ distance from the source. ${ }^{12}$ It is a combined uncertainty of source air-kerma strength, dose-rate constant and radial dose function.

\section{RESULTS}

\subsection{Detector-to-detector variation}

\subsubsection{Calibration data}

Calibration coefficients of microDiamond detectors and silicon diodes in terms of air-kerma free in air $N_{K \text {,air }}$ and absorbed dose to water $N_{D, \mathrm{w}, Q_{0}}$ in ${ }^{60} \mathrm{Co}$ beams shown in Figure 1 reveal the 
intra-detector variability of microDiamonds (see Table 1 for conversion of beam quality to a corresponding effective photon beam energy).

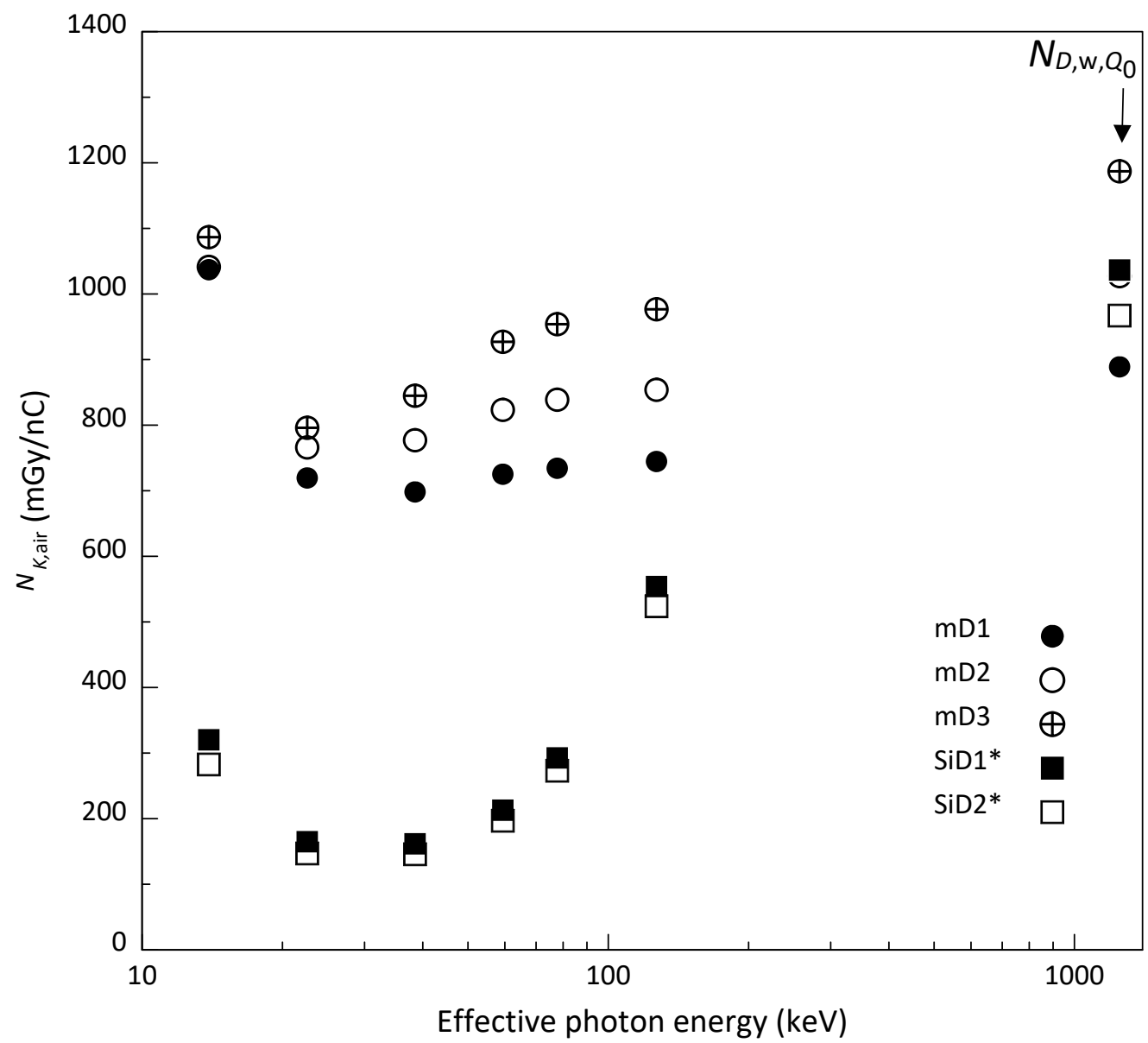

Figure 1 Calibration coefficients in terms of air-kerma free in air $\boldsymbol{N}_{\boldsymbol{K} \text {,air }}$ of all detectors as a function of the effective photon beam energy and in terms of the absorbed dose to water $\boldsymbol{N}_{\boldsymbol{D}, \mathbf{w}, \boldsymbol{Q}_{\mathbf{0}}}$ in ${ }^{60} \mathrm{Co}$ beam (1250 keV energy). Numerical values are provided in the supplementary material. *All SiD1 and SiD2 values were multiplied by ten. The bar size of the expanded uncertainty $(k=2)$ was comparable to the marker size and hence is not displayed.

Variation in $N_{D, \mathrm{w}, Q_{0}}$ of microDiamond detectors by $30 \%$ may be caused by different thicknesses of active volumes as described by Marinelli et al. ${ }^{31}$ However, it is evident from Figure 2, where $N_{K, \text { air }}$ was normalized to $N_{D, \mathrm{w}, Q_{0}}$ of a corresponding detector, that the difference in active volume alone could not explain the observed detector behavior at low energies where a proportionality between $N_{K, \text { air }}$ and $N_{D, \mathrm{w}, Q_{0}}$ no longer holds. 


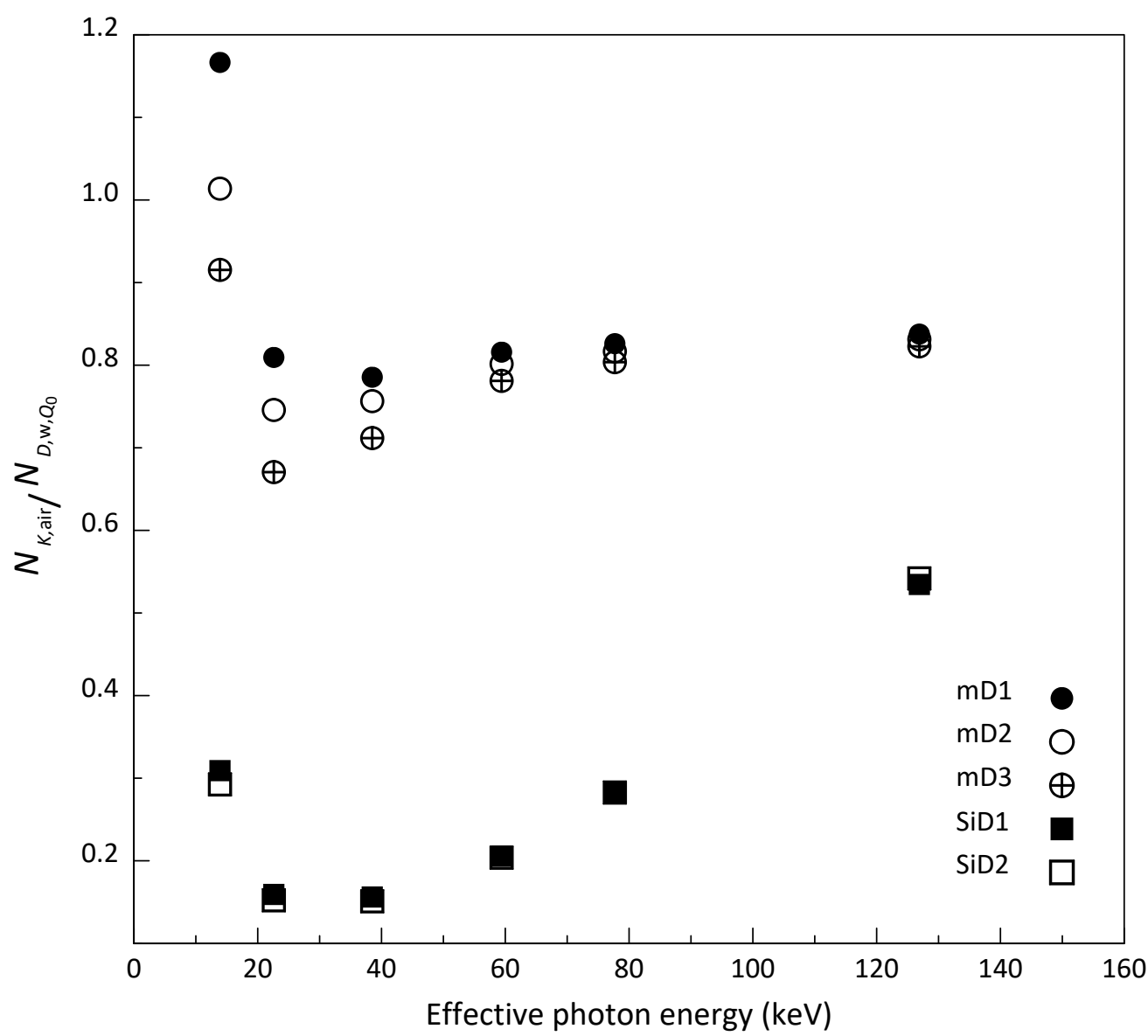

Figure 2 Calibration coefficients in terms of air-kerma free in air $\boldsymbol{N}_{\boldsymbol{K} \text {,air }}$ of all detectors normalized to their respective calibration coefficients in terms of the absorbed dose to water in ${ }^{60} \mathrm{Co}$ beam $\boldsymbol{N}_{\boldsymbol{D}, \mathbf{w}, \boldsymbol{Q}_{\mathbf{0}}}$ as a function of the effective photon beam energy. The bar size of the expanded uncertainty $(k=2)$ was comparable to the marker size and hence is not displayed.

Contrary to microDiamond detectors, it is interesting to point out that calibration coefficients $N_{D, \mathrm{w}, Q_{0}}$ of the silicon diodes differed by $7 \%$ in the calibration beam quality ${ }^{60} \mathrm{Co}$ compared with one another, and the difference in $N_{K \text {,air }}$ between the two detectors was approximately the same ( 7 to $13 \%$ ) over the entire $\mathrm{kV}$ energy range.

\subsubsection{MC simulations of effects associated with detector design}

\section{Size of the active volume}

Figure 3 shows that an increase in the active layer thickness from 1 to $2 \mu \mathrm{m}$ resulted in a decrease in the average absorbed doses to detectors by $7-8 \%$ in 25 and $50 \mathrm{kV}$ photon beams, 
$4 \%$ in the $100 \mathrm{kV}$ beam, and no statistically significant changes were observed for other $\mathrm{kV}$ beams. Poppinga et al. ${ }^{32}$ estimated the thickness of $2 \mu \mathrm{m}$ using a proton microbeam. They hypothesized that there is a diffusion zone in addition to the depletion layer where electronhole pairs are collected.

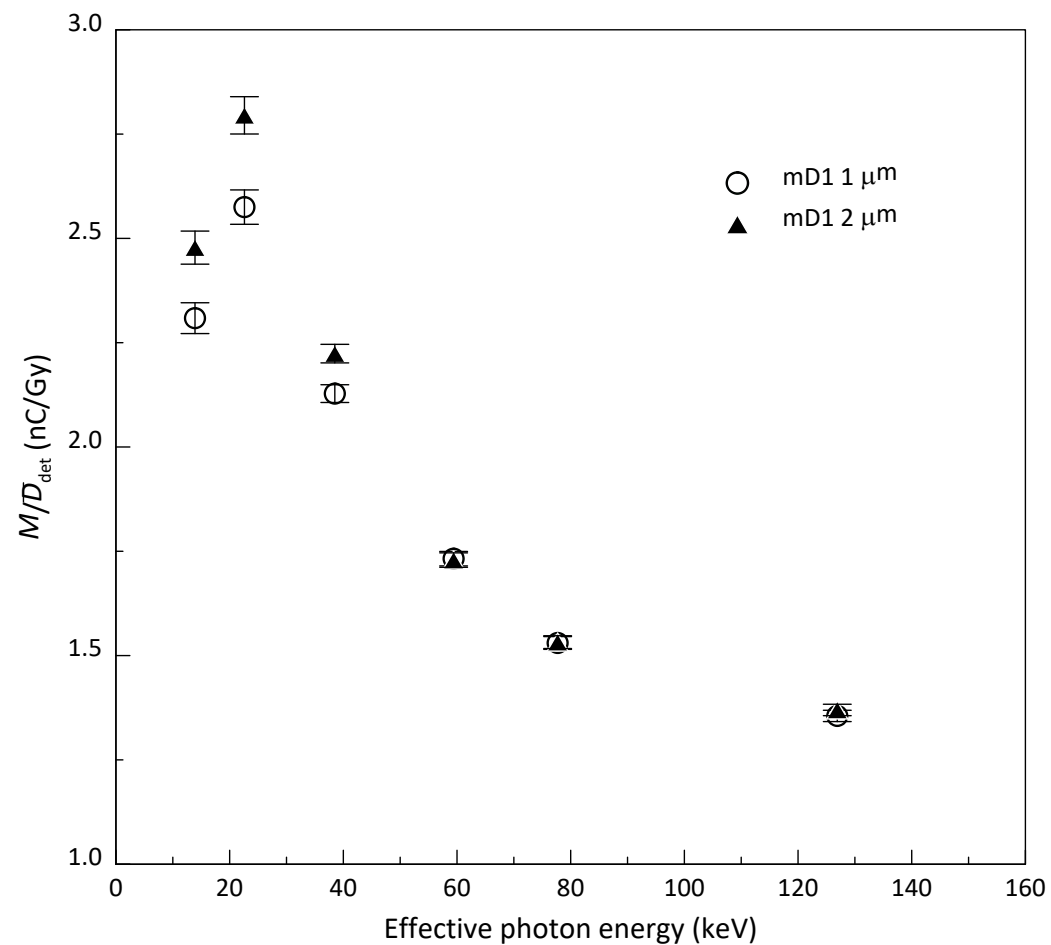

Figure 3 Intrinsic energy response of $\mathrm{mD} 1$ as a function of the effective photon beam energy. "mD1 $1 \mu \mathrm{m}$ " refers to the nominal detector model, "mD1 $2 \mu \mathrm{m}$ " has an active volume layer of $2 \mu \mathrm{m}$ thickness in MC simulations. The uncertainty bars correspond to the total expanded uncertainty in $\boldsymbol{R}_{\boldsymbol{Q}}(k=2)$.

This decrease in the average absorbed dose to the detector (an increase in $R=M / \bar{D}_{\text {det }}$ in Figure 3 ) is caused by a decrease in the average fluence of secondary electrons in a thicker active volume. When the thickness of the active volume is increased, low energy secondary electrons from the neighboring regions do not have enough energy to penetrate to the central region of the active volume and thus the average absorbed dose in it is decreased. Figure 4 shows that the distribution of fluence with respect to energy $\phi_{E}$ for secondary electrons produced by a $25 \mathrm{kV}$ photon beam peaks at an energy close to $10 \mathrm{keV}$. The continuous slowing down approximation range of $10 \mathrm{keV}$ electrons in diamond is approximately $0.8 \mu \mathrm{m}$. An increase in the thickness 
from 1 to $2 \mu \mathrm{m}$ thus decreased $\phi_{E}$ in the low-energy part of the spectrum and resulted in volume averaging effect (most of the electrons are absorbed in the first half of the active layer). No significant difference in either electron fluence spectrum or energy deposited per unit mass was found when we increased the nominal thickness of $30 \mu \mathrm{m}$ by $6 \%$ based on the difference between calibration coefficients $N_{D, \mathrm{w}, Q_{0}}$ of the two SiDs.

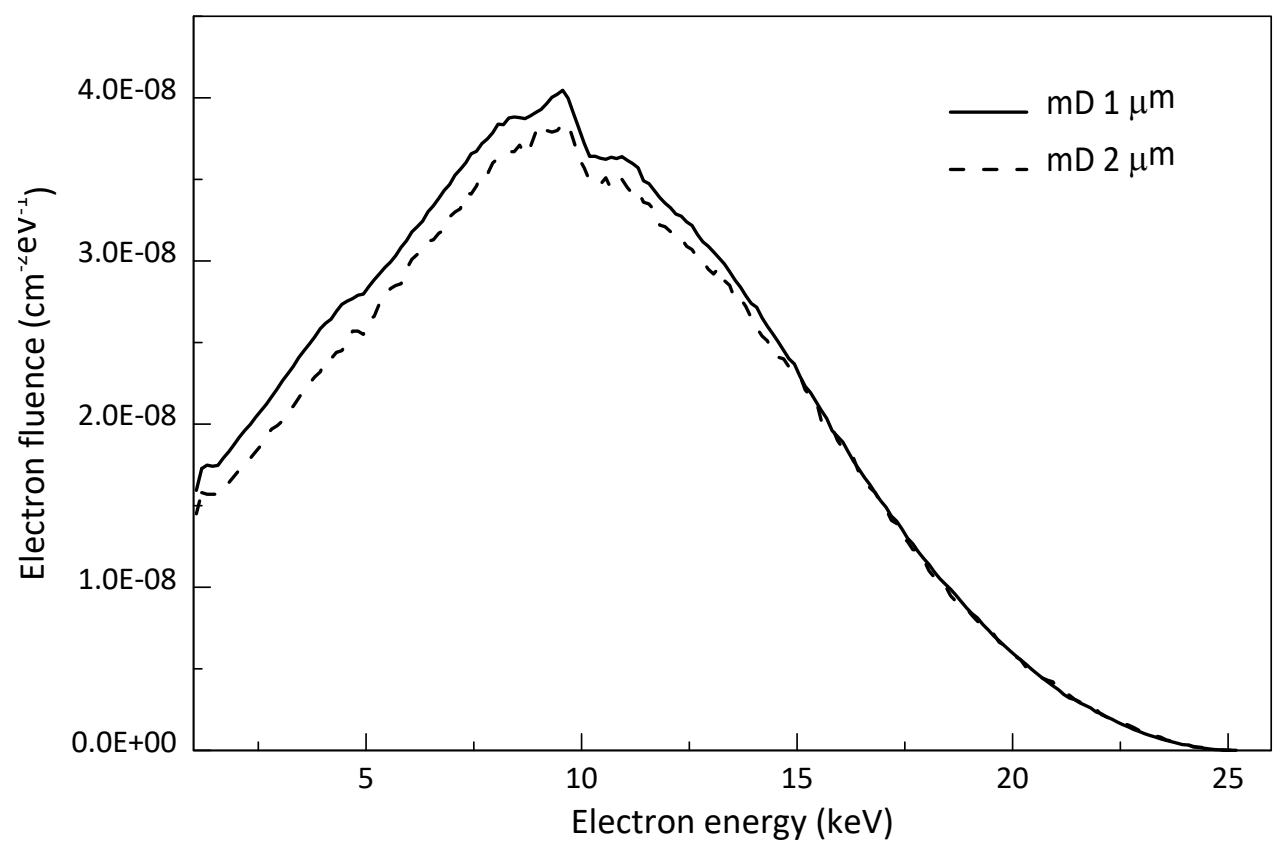

Figure 4 MC-calculated electron fluence spectra $\phi_{E}$ scored in the active volume of detectors as a function of secondary electron energy for a $25 \mathrm{kV}$ photon beam. "mD $1 \mu \mathrm{m}$ " refers to the detector model provided by the manufacturer, "mD $2 \mu \mathrm{m}$ " refers to the nominal model but with a $2 \mu \mathrm{m}$ thickness active layer.

\section{Extra-cameral components}

A replacement of all the metallic extra-cameral components with the resin-like encapsulation material decreased the energy imparted to the active volume by 10.7 and $2.5 \%$ in $25 \mathrm{kV}$ and $250 \mathrm{kV}$ photon beams respectively.

\subsection{Absorbed-dose energy response of detectors}

The MC-calculated absorbed-dose energy response of detectors with respect to air is shown in Figure 5 together with the two ideal cases of a large and a small cavity of bare active materials. 
In the first case, energy imparted to the cavity is due to secondary electrons produced inside the cavity under charged-particle equilibrium. For monoenergetic photons, the ratio $\bar{D}_{\text {det }} / K_{\text {air }}$ can be approximated as a ratio of mass-energy absorption coefficients $\left[\mu_{\mathrm{en}} / \rho\right]_{\mathrm{air}}^{\mathrm{det}}$. In the second case, no secondary electrons are produced in the cavity and the electron fluence of electrons produced outside the cavity is not perturbed by the presence of the cavity. Hence the ratio $\bar{D}_{\text {det }} / K_{\text {air }}$ can be approximated as a ratio of mass electronic stopping powers weighted over the energy distribution of the electron fluence $\left[\bar{S}_{\mathrm{el}} / \rho\right]_{\mathrm{air}}^{\mathrm{det}}$. Figure 5 shows mass electronic stopping power ratios calculated for monoenergetic electrons. Since the function varies little with energy, similar behavior is expected for the weighted mass electronic stopping power ratios.
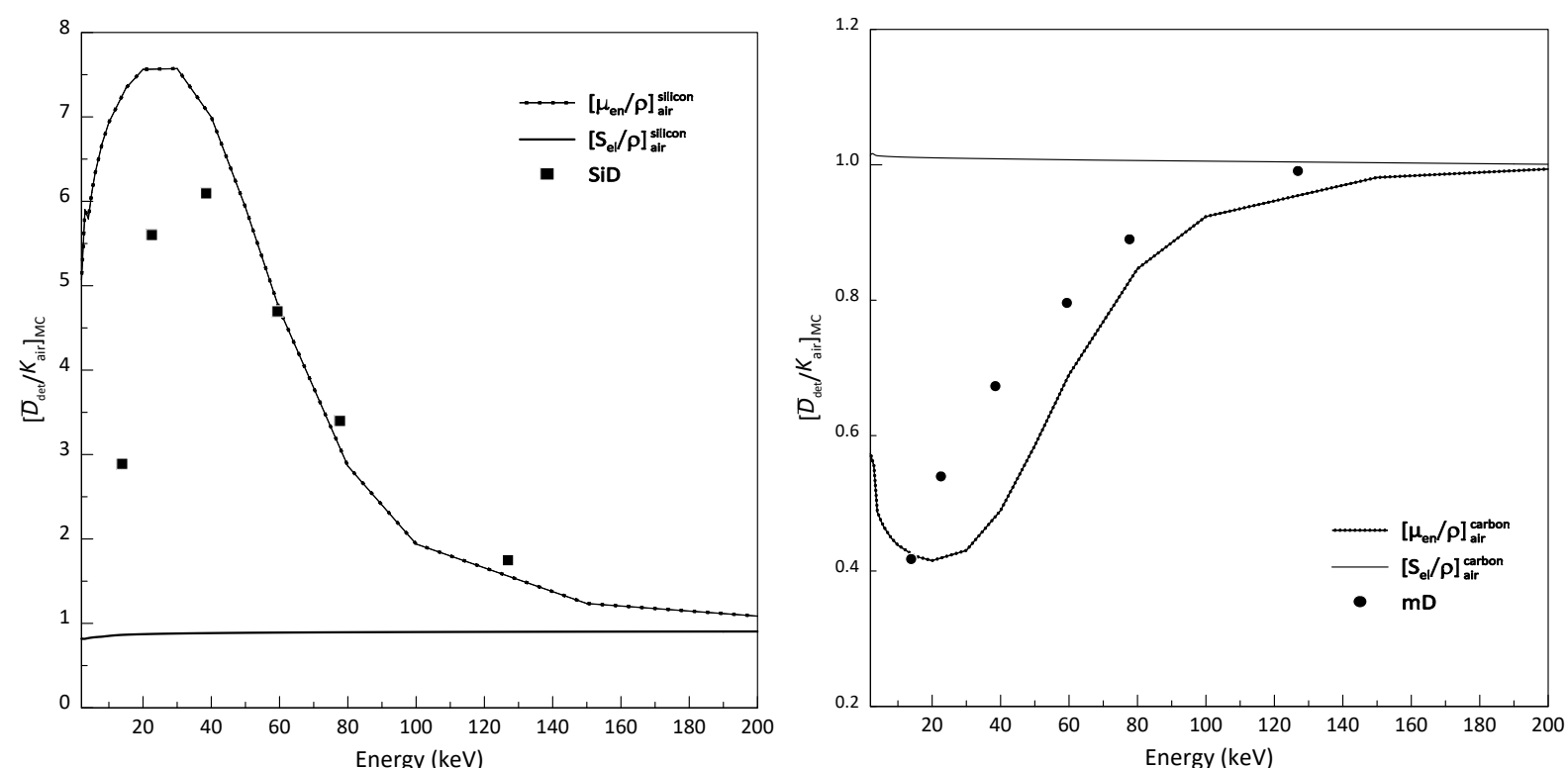

Figure $5 \mathrm{MC}$ calculated $\overline{\boldsymbol{D}}_{\text {det }} / \boldsymbol{K}_{\text {air }}$ ratios as a function of the effective photon beam energy (see Table 1) for silicon diode (left) and microDiamond (right) detectors used in the study. Solid and dotted lines represent the ratio of mass electronic stopping powers $\left[\boldsymbol{S}_{\mathrm{el}} / \boldsymbol{\rho}\right]_{\text {air }}^{\mathrm{det}}$ and mass energy-absorption coefficients $\left[\boldsymbol{\mu}_{\mathrm{en}} / \boldsymbol{\rho}\right]_{\text {air }}^{\mathrm{det}}$ respectively of the detector cavities to air for monoenergetic beams.

Although the absorbed-dose energy dependence of both silicon diode and microDiamond detectors was closer to the large-cavity case, none of the ideal cases were fulfilled. The detector perturbs the radiation field at the point of measurement compared to a configuration without the detector. Additionally, electron absorption on the spot could no longer be assumed for the 
microDiamond detectors due to small thicknesses of the active volumes compared to the electron range. For instance, approximately $40 \%$ of the energy deposited in the active volume in $25 \mathrm{kV}$ photon beam was due to electrons entering from outside the cavity.

\subsection{Intrinsic energy response of detectors}

The determined intrinsic energy response of microDiamond detectors, i.e., the detector signal per average absorbed dose to the detector $\left(R=M / \bar{D}_{\text {det }}\right)$, substantially increases with decreasing photon energy compared to that in the ${ }^{60} \mathrm{Co}$ beam as shown in Figure 6.

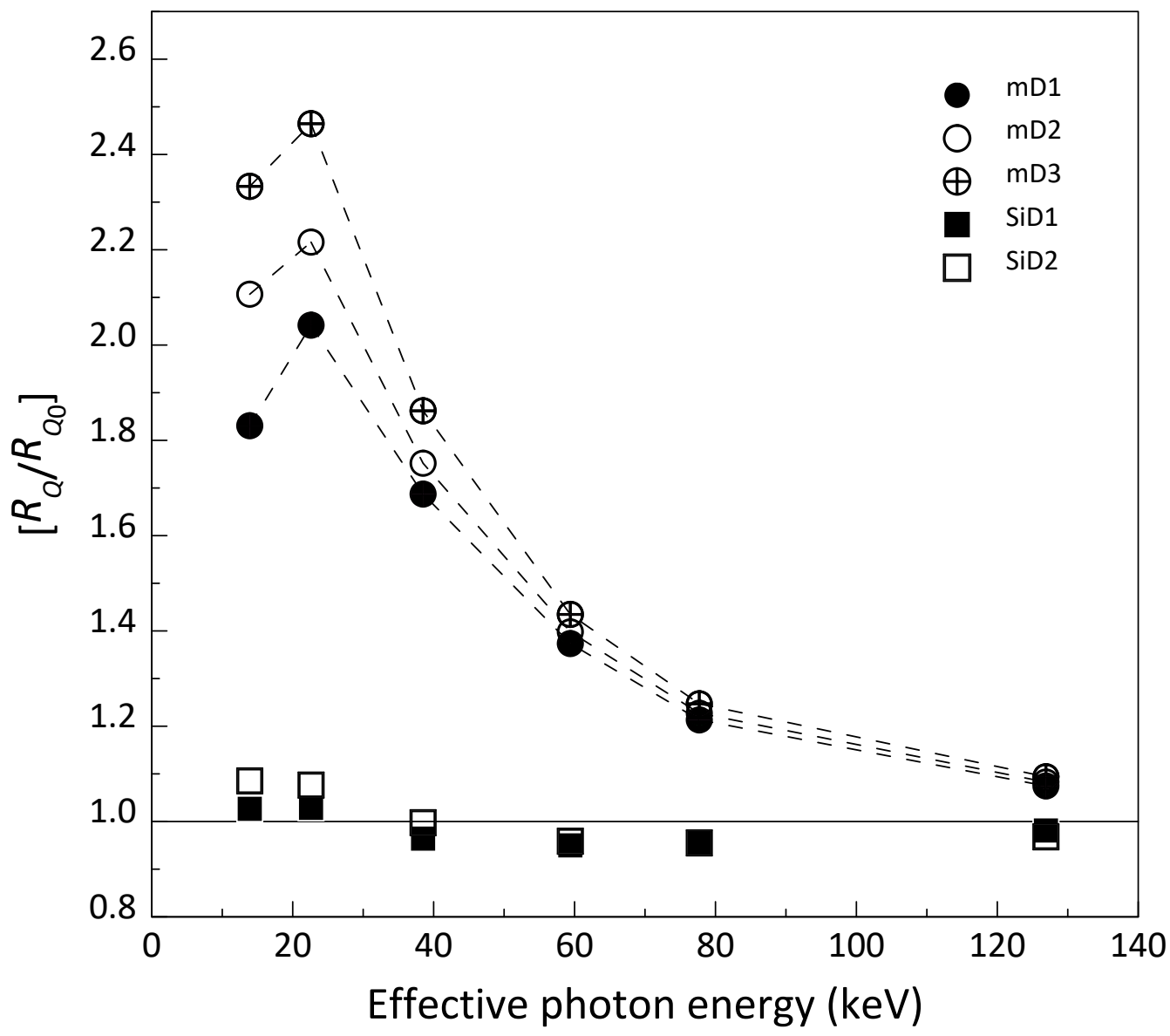

Figure 6 The relative intrinsic energy response of microDiamond and silicon diode detectors as a function of the effective photon beam energy (here $\boldsymbol{Q}$ is the $\mathrm{kV}$ calibration beam quality and $\boldsymbol{Q}_{\mathbf{0}}$ is the ${ }^{60} \mathrm{Co}$ calibration beam). Numerical values are provided in the supplementary material. Dashed lines are for visual guidance. The bar size of the expanded uncertainty $(k=2)$ was comparable to the marker size and hence is not displayed. 
Compared to detector response in ${ }^{60} \mathrm{Co}$ beam, the detector is approximately twice as efficient in 25 and $50 \mathrm{kV}$ photon beams, i.e, approximately 1.8-2.5 nC/Gy versus $1 \mathrm{nC} / \mathrm{Gy}$. The intrinsic energy dependence of the two silicon diodes did not vary by more than $10 \%$ over the same energy range. Since we extracted the intrinsic energy response of detectors from their calibration coefficients $N_{K \text {,air }}$ and $N_{D, \mathrm{w}, Q_{0}}$, any intra-detector variation discussed in Section 3.1 is seen in these results.

\subsection{Measurements with an ${ }^{125}$ I BT seed}

The applicability of the relative intrinsic energy response $R_{Q_{0}} / R_{Q}$ correction factors was checked for an ${ }^{125}$ I BT seed in an independent setup. Experimentally-determined and TPScalculated absorbed dose to water values $D_{\mathrm{w}}$ in ${ }^{125} \mathrm{I}$ beam are shown in Table 4 .

Table 4 Experimentally-determined absorbed dose to water $\boldsymbol{D}_{\mathbf{w}}$ values for 5 and $10 \mathrm{~mm}$ source-to-detector distances (SDDs). For comparison, treatment planning system (TPS) calculated values are also shown.

\begin{tabular}{cccccc}
\hline \hline SDD (mm) & TPS (mGy) & mD1 (mGy) & mD2 (mGy) & mD3 (mGy) & SiD2 (mGy) \\
\hline \hline 5 & $5.9 \pm 0.7$ & $6.2 \pm 0.6$ & $5.9 \pm 0.5$ & $5.8 \pm 0.5$ & $4.4 \pm 0.4$ \\
10 & $1.7 \pm 0.2$ & $1.3 \pm 0.1$ & $1.4 \pm 0.1$ & $1.3 \pm 0.1$ & $1.3 \pm 0.1$ \\
\hline \hline
\end{tabular}

$D_{\mathrm{w}}$ values calculated using Eq. (1.7) agree within the experimental uncertainties among all the detectors for both SDDs. The $R_{Q_{0}} / R_{Q}$ values used for ${ }^{125}$ I beam were interpolated from Figure 6 and were from 0.45 to 0.51 for the three microDiamond detectors and 0.96 for SiD2. The remaining discrepancy of $5-30 \%$ from the TPS could be partly due to the use of correction factors derived using broad $\mathrm{kV}$ spectra, whereas ${ }^{125} \mathrm{I}$ can be considered as a monoenergetic source. Furthermore, irradiation geometries differed in the two setups. Given that, the comparison with the TPS should be taken more as a qualitative check of the calculated relative intrinsic energy response ratios. 


\section{DISCUSSION}

\subsection{Detector-to-detector variation}

\subsubsection{Calibration data}

Results in Figure 2 indicate that microDiamond intra-detector variability is affected by factors other than detector active volume size; either related to their energy absorption properties, e.g., differences in their construction affecting their water-equivalence or related to solid state physics and charge collection. The scope of this paper was to extract the intrinsic energy response of microDiamond detectors using the MC-calculated absorbed-dose energy response. However, as shown in several studies on synthetic diamond detectors the response may also depend on other factors. For instance, changes in charge collection due to non-conformity of electric field in the detector, ${ }^{33}$ the presence of impurities, ${ }^{34}$ higher responsivity to low-energy $\mathrm{x}^{- \text {rays }}{ }^{35}$ and field-size dependent charge imbalance in the structural components of the detector. $^{36}$ The latter effect explained discrepancies between measurements and $\mathrm{MC}$ simulations in $6 \mathrm{MV}$ small fields, but it cannot explain the intra-detector variability reported in this article.

There have been indications that the homogeneity of microDiamond detector response could be energy-dependent and affect detectors to a different extent. Local and gradual variations from 15 to $30 \%$ were observed in microDiamond detector response (homogeneity) when detectors were irradiated with a narrow synchrotron beam (the weighted average energy of $95 \mathrm{keV}$ ) whose impact position was varied over the active surface of the detector. ${ }^{37}$ On the other hand, a lower average homogeneity of $3.6 \%$ was reported for diamonds studied in a $35 \mathrm{kV}$ photon beam. ${ }^{31}$ The microDiamond detectors used in our study were most likely also affected by such non-uniform response which contributed to the intra-detector variation and its dependence on beam quality. No such non-uniformity in response was observed for other investigated 
detectors, e.g., PTW 60017 silicon diodes. ${ }^{37}$ This agrees with our results where differences in $N_{K, \text { air }}$ between the two investigated PTW 60017 silicon diodes could be explained by differences in $N_{D, \mathrm{w}, Q_{0}}$ calibration coefficients alone (Figure 2), i.e., a difference in the active volume size.

\subsubsection{MC simulations of effects associated with detector design}

Change in detector active volume thickness could account for microDiamond intra-detector variability by up to $8 \%$ at the lowest energy $\mathrm{kV}$ spectra. Results of extra-cameral component influence on detector response complement the experimental findings by Butler et al. ${ }^{37}$ where it is estimated that approximately $8 \%$ of the measured signal is produced in components other than the active volume when the detector is irradiated from a side with a narrow synchrotron beam. It must be noted that we determined the perturbation caused by non-water equivalent extra-cameral components which affect energy deposition in the active volume (hence the absorbed-dose energy response of the detector), whereas Butler et al. ${ }^{37}$ hypothesize that the measured extra signal could be due to charge induced and collected in the extra-cameral components not reaching the active volume.

\subsection{Intrinsic energy response of detector}

The reason for a large variation in the intrinsic energy response of microDiamond could be that physical electron-hole pair generation and collection processes, such as charge recombination, trapping, diffusion from nearby layers into the active volume, were beam quality dependent. Nevertheless, we must emphasize that the intrinsic energy response is extracted from the overall response of the detectors using MC calculations (see Eq. (1.5)), and hence it relies highly on detector model accuracy. Any mismatch between the actual detector model and blueprints would affect the magnitude of MC-calculated absorbed-dose energy response values, and subsequently, the shape of the intrinsic energy response function in Figure 6. For example, the 
presence of any high- $Z$ element contamination near the active volume may result in larger values of the absorbed dose to detector at low energies, and that would reduce $R_{Q} / R_{Q_{0}}$ values. The observed decrease in $R_{Q} / R_{Q_{0}}$ at the effective photon energy of $13.9 \mathrm{keV}(25 \mathrm{kV}$ beam spectrum) could be due to detector under-response when the linear energy transfer (LET) of secondary electrons increases. An under-response of a microDiamond was reported in highLET carbon and oxygen beams, ${ }^{7}$ but in proton beams the reported results were inconclusive indicating either no significant LET dependence ${ }^{7,8}$ or under-response. ${ }^{6}$

The $R_{Q} / R_{Q_{0}}$ values are higher only at the lowest energies and approach unity with increasing energy. Our results hence do not contradict findings in high-energy photon and electron beams reporting negligible beam quality dependence. ${ }^{1,38,39}$ Furthermore, no significant relative intrinsic energy dependence was observed in our previous study ${ }^{9}$ of microDiamond detectors in ${ }^{192}$ Ir beam which had an effective photon energy close to $300 \mathrm{keV}$.

\subsection{Measurements with an ${ }^{125}$ I seed}

It is evident that the use of the relative intrinsic energy response correction factors improved the agreement between both types of detectors and with the TPS calculated $D_{\mathrm{w}}$ values. Without them, the microDiamond detectors would overestimate absorbed dose to water by almost a factor of two compared to the TPS.

Figure 7 shows ratios of SiD2 and $\mathrm{mD} 1$ detector readings in ${ }^{125} \mathrm{I}$ beam at $5 \mathrm{~mm}$ and $10 \mathrm{~mm}$ distance from the source and in calibration beams. For comparison, the corresponding detector reading ratios in the ${ }^{192} \mathrm{Ir}$ beam for three SDDs $(15,25$ and $55 \mathrm{~mm})$ are included from a previous study. ${ }^{9}$ If the detectors' response would be affected only by their absorbed-dose energy response (Section 3.2), the change in detector reading-ratio per absorbed dose to water or air-kerma (depending on measurement setup) would be approximately equal to MC-calculated ratios $\bar{D}_{\mathrm{SiD}} / \bar{D}_{\mathrm{mD}}$ or a given beam quality (see insert in Figure 7). The shape of detector reading ratios 
and MC-calculated $\bar{D}_{\mathrm{SiD}} / \bar{D}_{\mathrm{mD}}$ ratios as a function of photon energy is approximately the same but the difference in magnitude indicates the presence of intrinsic energy response. Most importantly, the data from experimental measurements in ${ }^{125} \mathrm{I}$ and ${ }^{192} \mathrm{Ir}$ beams agree well with the data from calibration beams traceable to the primary standards.

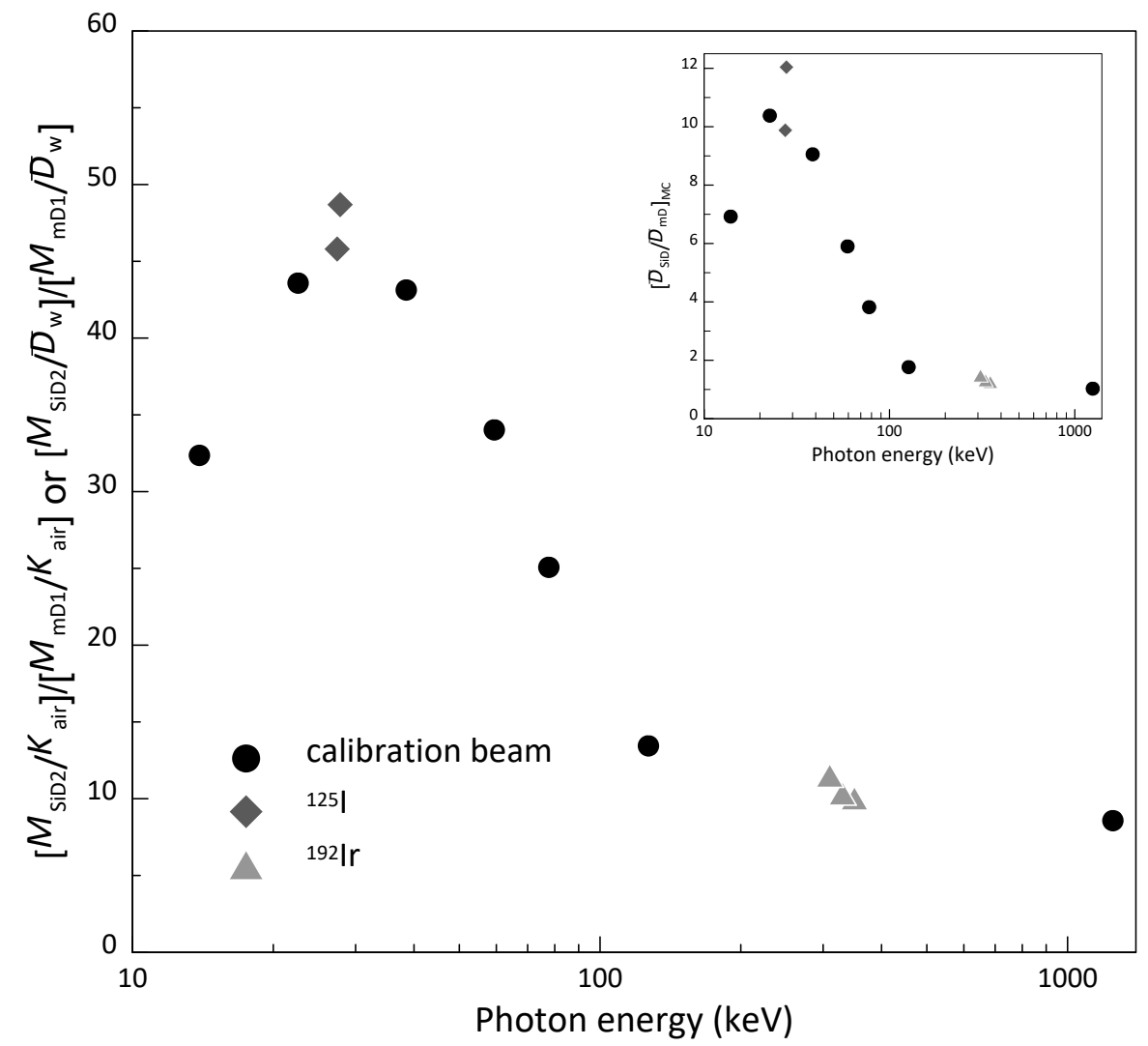

Figure 7 The ratio of SiD2 and mD1 detector readings per absorbed dose to water (in ${ }^{125} \mathrm{I},{ }^{192} \mathrm{Ir}$ and ${ }^{60} \mathrm{Co}$ beams) and per air-kerma ( $\mathrm{kV}$ calibration beams) as a function of the effective photon beam energy. Data for ${ }^{125} \mathrm{I}$ and ${ }^{192} \mathrm{Ir}$ beams is shown for different source-to-detector distances corresponding to different photon fluence-weighted energies. Data points for ${ }^{192}$ Ir beam were taken from Kaveckyte et al. ${ }^{9}$ Insert: The MC-calculated ratio of average absorbed dose to $\mathrm{SiD}$ and $\mathrm{mD}$ for the corresponding beam qualities. All data points are normalized to ${ }^{60} \mathrm{Co}$ beam. The bar size of the expanded uncertainty $(k=2)$ was comparable to the marker size and hence is not displayed.

Since the measurement setup with an ${ }^{125}$ I BT seed and simulation input files were completely independent from those used in $\mathrm{kV}$ calibration measurements and simulations (apart from blueprints), such a comparison serves as a qualitative validation of our current data on the intrinsic energy dependence of detectors presented in Figure 6. 


\subsection{Uncertainty analysis}

The presented model uses a large number of input parameters describing cross sections, materials and geometry. Though reasonable assumptions on uncertainties of these parameters can be made, covariances between these parameters required by the "Guide to the expression of uncertainty in measurement" are difficult to estimate. Also, the analysis requires multiple runs of the CPU-time demanding code to properly sample the input parameter space. A fullscale analysis may be the subject of a future work, but currently, it is beyond the time and resources of the authors. For these reasons, only rough estimates of upper bounds of uncertainty are provided in the following text.

Cross sections in the studied energy range are dominated by photoelectric effect (PE). An increase by $2 \%$ in the PE cross sections of all considered materials lead to an increase by $1.4 \%$ and $2.3 \%$ in energy deposited in the active volume of microDiamond for the $25 \mathrm{kV}$ and ${ }^{125} \mathrm{I}$ sources, respectively. Absorbed dose to water at a point of measurement in the absence of the detector increased by $1.6 \%$ in ${ }^{125} \mathrm{I}$ beam. Since the quantity used to determine the intrinsic energy response of detectors is the ratio of $D_{\mathrm{w}}$ and $\bar{D}_{\mathrm{det}}$, the resulting effect depends on the correlation between $D_{\mathrm{w}}$ and $\bar{D}_{\text {det }}$; a positive correlation will lead to a decreased effect, a negative correlation will lead to an increased effect. Thus, it is reasonable to assume that the uncertainty arising from uncertainty in cross section data is on the order of a few percent.

Uncertainties in the PMMA phantom composition and density and their effect on the MCcalculated absorbed dose to detector active volume in the PMMA phantom $\left(\bar{D}_{\text {det }}\right)$ were not evaluated. Rodriguez et al..$^{40}$ estimated that an uncertainty in solid water composition and density would result in a 3\% uncertainty in MC-calculated quantities in ${ }^{125} \mathrm{I}$ BT beams. In contrast to solid water or RW3 plastic phantoms, which can have varying amounts of calcium and titanium dioxide additives, ${ }^{40-42}$ phantoms made of PMMA have more uniform and better- 
known composition. ${ }^{12,43}$ Thus it is reasonable to assume that the uncertainty associated with material composition is on the order of a few percent.

\section{CONCLUSIONS}

In this study we found that the intrinsic energy response of microDiamond detectors increased by a factor of two at the lowest energy photon beams compared to that in a ${ }^{60} \mathrm{Co}$ beam. Moreover, the overall energy response varied by up to $30 \%$ among the three investigated microDiamond detectors in low energy photon beams. The significant relative intrinsic energy dependence implies that the generic beam quality correction factors calculated using $\mathrm{MC}$ are not enough for use in low energy photon beams when detectors are calibrated in high energy photon beams as it is commonly done and recommended in the BT TG-43 dosimetry protocol. ${ }^{12}$ Silicon diode detectors, which have a pronounced absorbed-dose energy dependence compared to air and water, have an intrinsic energy dependence similar to that of passive readout detectors studied elsewhere, i.e., deviating from unity by up to $5 \%$.

These findings show that it is important to determine both the absorbed-dose and the intrinsic energy responses for certain types of detectors and beam qualities. Though the results and analysis of this study are very dependent on the MC model used to model absorbed-dose energy response of detectors, detector blueprints were as accurate as possible according to the manufacturer. The cause of the large variation of the relative intrinsic energy response of the microDiamond detector with respect to energy, however, is yet to be determined.

\section{ACKNOWLEDGEMENTS}

Grants from the Swedish Cancer Society (Cancerfonden) (CAN 2015/618 and CAN 2018/622) are acknowledged. We would also like to thank Dr. J. Wuerfel from PTW-Freiburg for helpful discussions regarding the construction of a microDiamond detector. 


\section{CONFLICTS OF INTEREST}

The authors have no relevant conflicts of interest to disclose.

\section{REFERENCES}

1. Laub WU, Crilly R. Clinical radiation therapy measurements with a new commercial synthetic single crystal diamond detector. J Appl Clin Med Phys. 2014;15(6):1-4.

2. Ralston A, Tyler M, Liu P, McKenzie D, Suchowerska N. Over-response of synthetic microDiamond detectors in small radiation fields. Phys Med Biol. 2014;59(19):5873-5881.

3. Di Venanzio C, Marinelli M, Tonnetti A, et al. Characterization of a microDiamond detector in high-dose-per-pulse electron beams for intra operative radiation therapy. Physica Medica. 2015;31(8):897-902.

4. De Coste V, Francescon P, Marinelli M, et al. Is the PTW 60019 microDiamond a suitable candidate for small field reference dosimetry? Phys Med Biol. 2017;62(17):7036-7055.

5. Marinelli M, Prestopino G, Verona C, et al. Dosimetric characterization of a microDiamond detector in clinical scanned carbon ion beams. Med Phys. 2015;42(4):2085-2093.

6. Marsolat F, De Marzi L, Patriarca A, et al. Dosimetric characteristics of four PTW microDiamond detectors in high-energy proton beams. Phys Med Biol. 2016;61(17):6413-6429.

7. Rossomme S, Hopfgartner J, Vynckier S, Palmans H. Under-response of a PTW-60019 microDiamond detector in the Bragg peak of a $62 \mathrm{MeV} / \mathrm{n}$ carbon ion beam. Phys Med Biol. 2016;61(12):4551-4563.

8. Goma C, Marinelli M, Safai S, Verona-Rinati G, Wurfel J. The role of a microDiamond detector in the dosimetry of proton pencil beams. Z Med Phys. 2016;26(1):88-94.

9. Kaveckyte V, Malusek A, Benmakhlouf H, Alm Carlsson G, Carlsson Tedgren A. Suitability of microDiamond detectors for the determination of absorbed dose to water around high-doserate 192Ir brachytherapy sources. Med Phys. 2018;45(1):429-437.

10. Rossi G, Gainey M, Thomann B, et al. (in press) Monte Carlo and experimental high dose rate ${ }^{192} \mathrm{Ir}$ brachytherapy dosimetry with microDiamond detectors. Z Med Phys. 2018:doi: 10.1016/j.zemedi.2018.1009.1003.

11. Damodar J, Odgers D, Pope D, Hill R. A study on the suitability of the PTW microDiamond detector for kilovoltage x-ray beam dosimetry. Appl Radiat Isot. 2018;135:104-109.

12. Rivard MJ, Coursey BM, DeWerd LA, et al. Update of AAPM Task Group No. 43 Report: A revised AAPM protocol for brachytherapy dose calculations. Med Phys. 2004;31(3):633-674.

13. Rivard MJ, Ballester F, Butler WM, et al. Supplement 2 for the 2004 update of the AAPM Task Group No. 43 Report: Joint recommendations by the AAPM and GEC-ESTRO. Med Phys. 2017;44(9):297-337.

14. Almond PR, Biggs PJ, Coursey BM, et al. AAPM's TG-51 protocol for clinical reference dosimetry of high-energy photon and electron beams. Med Phys. 1999;26(9):1847-1870.

15. Andreo P, Burns DT, Hohlfeld K, et al. Absorbed dose determination in external beam radiotherapy: An international Code of Practice for dosimetry based on standards of absorbed dose to water. Vienna, Austria: International Atomic Energy Agency;2000.

16. Davis SD, Ross CK, Mobit PN, Van der Zwan L, Chase WJ, Shortt KR. The response of LiF thermoluminescence dosemeters to photon beams in the energy range from $30 \mathrm{kV} x$ rays to ${ }^{60}$ Co gamma rays. Radiat Prot Dosimetry. 2003;106(1):33-43.

17. Nunn AA, Davis SD, Micka JA, DeWerd LA. LiF:Mg,Ti TLD response as a function of photon energy for moderately filtered $\mathrm{x}$-ray spectra in the range of $20-250 \mathrm{kVp}$ relative to ${ }^{60} \mathrm{Co}$. Med Phys. 2008;35(5):1859-1869. 
18. Carlsson Tedgren A, Hedman A, Grindborg JE, Carlsson GA. Response of LiF:Mg,Ti thermoluminescent dosimeters at photon energies relevant to the dosimetry of brachytherapy (<1 MeV). Med Phys. 2011;38(10):5539-5550.

19. Hashimoto S, Nakajima Y, Kadoya N, Abe K, Karasawa K. Energy dependence of a radiophotoluminescent glass dosimeter for HDR ${ }^{192} \mathrm{Ir}$ brachytherapy source. Med Phys. 2018;46(2):964-972.

20. Adolfsson E, Alm Carlsson G, Grindborg JE, Gustafsson H, Lund E, Carlsson Tedgren A. Response of lithium formate EPR dosimeters at photon energies relevant to the dosimetry of brachytherapy. Med Phys. 2010;37(9):4946-4959.

21. Anton $M$, Buermann $L$. Relative response of the alanine dosimeter to medium energy $\mathrm{x}$-rays. Phys Med Biol. 2015;60(15):6113-6129.

22. Kessler C, Burns DT. Measuring conditions and unceratinties for the comparison and calibration of national dosimetric standards at the BIPM 2018.

23. Sempau J, Badal A, Brualla L. A PENELOPE-based system for the automated Monte Carlo simulation of clinacs and voxalized geometries-application to far-from-axis fields. Med Phys. 2011;38(11):5887-5895.

24. Salvat F. PENELOPE: a code system for Monte Carlo simulation of electron and photon transport. In: Issy-les-Moulineaux: OECD Nuclear Energy Agency; 2014.

25. Ribberfors R. X-ray incoherent scattering total cross sections and energy-absoprtion cross sections by means of simple calculation routines. Phys Rev A. 1983;27:3061-3070.

26. Cullen DE, Hubbell JH, Kissel L. EPDL97 The Evaluated Photon Data Library '97 Version. Livermore, California: Lawrence Livermore National Laboratory;1997.

27. Tables of $X$-ray mass attenuation coefficients and mass energy-absorption coefficients from 1 $\mathrm{keV}$ to $20 \mathrm{MeV}$ for elements $\mathrm{Z}=1$ to 92 and 48 additional substances of dosimetric interest National Institute of Standards and Technology, Gaithersburg, MD; 2004. http://www.nist.gov/pml/data/xraycoef/. Accessed January 15, 2019.

28. Andreo P, Burns DT, Salvat F. On the uncertainties of photon mass energy-absorption coefficients and their ratios for radiation dosimetry. Phys Med Biol. 2012;57(8):2117-2136.

29. Hedtjarn H, Carlsson GA, Williamson JF. Monte Carlo-aided dosimetry of the Symmetra model 125.S06 125I, interstitial brachytherapy seed. Med Phys. 2000;27(2):1076-1085.

30. JCGM. Evaluation of measurement data-guide to the expression of uncertainty in measurement (GUM). Sévres-BIPM Joint Committee for Guides in Metrology WG1;2008.

31. Marinelli M, Prestopino G, Verona C, Verona-Rinati G. Experimental determination of the PTW 60019 microDiamond dosimeter active area and volume. Med Phys. 2016;43(9):5205-5212.

32. Poppinga D, Delfs B, Meyners J, et al. Determination of the active volumes of solid-state photon-beam dosimetry detectors using the PTB proton microbeam. Med Phys. 2018;45(7):3340-3348.

33. Marsolat $\mathrm{F}$, Tromson $\mathrm{D}$, Tranchant $\mathrm{N}$, et al. Why diamond dimensions and electrode geometry are crucial for small photon beam dosimetry. J Appl Phys. 2015;118(23):234507.

34. Ade N, Nam TL, Mhlanga SH. An evaluation of some pertinent parameters that influence the dosimetric performance of synthetic diamond detectors. Radiat Phys Chem. 2013;86:42-51.

35. Almaviva S, Marinelli M, Milani E, et al. Chemical vapor deposition diamond based multilayered radiation detector: Physical analysis of detection properties. J Appl Phys. 2010;107(1):014511.

36. Looe HK, Poppinga D, Kranzer R, et al. The role of radiation-induced charge imbalance on the dose-response of a commercial synthetic diamond detector in small field dosimetry. Med Phys. 2019;46(6):2752-2759.

37. Butler DJ, Beveridge T, Lehmann J, Oliver CP, Stevenson AW, Livingstone J. Spatial response of synthetic microDiamond and diode detectors measured with kilovoltage synchrotron radiation. Med Phys. 2018;45(2):943-952. 
38. Akino Y, Gautam A, Coutinho L, Wurfel J, Das IJ. Characterization of a new commercial single crystal diamond detector for photon- and proton-beam dosimetry. I Radiat Res. 2015;56(6):912-918.

39. Brualla-González L, Gómez F, Pombar M, Pardo-Montero J. Dose rate dependence of the PTW 60019 microDiamond detector in high dose-per-pulse pulsed beams. Phys Med Biol. 2016;61(1):N11-N19.

40. Rodriguez M, Rogers DW. Effect of improved TLD dosimetry on the determination of dose rate constants for ${ }^{125}$ and ${ }^{103}$ Pd brachytherapy seeds. Med Phys. 2014;41(11):114301-114315.

41. Patel NS, Chiu-Tsao ST, Williamson JF, et al. Thermoluminescent dosimetry of the Symmetra 125 I model I25.S06 interstitial brachytherapy seed. Med Phys. 2001;28(8):1761-1769.

42. Schoenfeld AA, Harder D, Poppe B, Chofor N. Water equivalent phantom materials for (1)(9)(2)Ir brachytherapy. Phys Med Biol. 2015;60(24):9403-9420.

43. Fulkerson RK, Micka JA, DeWerd LA. Dosimetric characterization and output verification for conical brachytherapy surface applicators. Part I. Electronic brachytherapy source. Med Phys. 2014;41(2):022103. 
Numerical values of data shown in Figure 1 are given in Table S-1.

Table S-1 Detector calibration coefficients in terms of air-kerma free in air $N_{K \text {,air }}$ and in terms of absorbed dose to water $N_{D, \mathrm{w}, Q_{0}}$. Effective energy for $\mathrm{kV}$ beam spectra was calculated as the energy of a monoenergetic beam which would have the same half-value layer as a given spectrum.

\begin{tabular}{|c|c|c|c|c|c|c|c|}
\hline Beam & $\begin{array}{c}\text { Generating } \\
\text { potential }(\mathrm{kV}) \\
\end{array}$ & $\begin{array}{c}\text { Effective energy } \\
(\mathrm{keV})\end{array}$ & $\mathrm{mD} 1$ & $\mathrm{mD} 2$ & $\mathrm{mD} 3$ & SiD1 & SiD2 \\
\hline & & & \multicolumn{5}{|c|}{$N_{K, \text { air }}(\mathrm{mGy} / \mathrm{nC})$} \\
\hline CCRI-25 & 25 & 13.9 & 1037.0 & 1041.4 & 1086.8 & 32.1 & 28.3 \\
\hline CCRI-50(b) & 50 & 22.6 & 719.5 & 766.1 & 796.1 & 16.5 & 14.7 \\
\hline CCRI-100 & 100 & 38.5 & 698.2 & 777.1 & 845.0 & 16.2 & 14.6 \\
\hline CCRI-135 & 135 & 59.4 & 725.2 & 823.4 & 927.3 & 21.3 & 19.7 \\
\hline CCRI-180 & 180 & 77.7 & 734.3 & 838.9 & 954.2 & 29.3 & 27.3 \\
\hline \multirow[t]{2}{*}{ CCRI-250 } & 250 & 126.9 & 744.6 & 854.0 & 976.9 & 55.4 & 52.4 \\
\hline & & & \multicolumn{5}{|c|}{$N_{D, \mathrm{w}, Q_{0}}(\mathrm{mGy} / \mathrm{nC})$} \\
\hline${ }^{60} \mathrm{Co}$ & & 1250 & 889.0 & 1027.3 & 1187.3 & 103.7 & 96.7 \\
\hline
\end{tabular}


Numerical values of data shown in Figure 6 are given in Table S-2.

Table S-2 The relative intrinsic energy response $R_{Q} / R_{Q_{0}}$ values of detectors as a function of the effective photon beam energy ( $Q$ is the $\mathrm{kV}$ calibration beam quality and $Q_{0}$ is the ${ }^{60} \mathrm{Co}$ calibration beam). The total expanded uncertainties $(k=2)$ were $2.0 \%$ for 25 and $50 \mathrm{kV}$ beams and $1.9 \%$ for other beams.

\begin{tabular}{cccccccc}
\hline \hline \multirow{2}{*}{ Beam } & \multirow{2}{*}{$\begin{array}{c}\text { Generating } \\
\text { potential }(\mathrm{kV})\end{array}$} & \multirow{2}{*}{$\begin{array}{c}\text { Effective energy } \\
(\mathrm{keV})\end{array}$} & \multicolumn{5}{c}{$R_{Q} / R_{Q_{0}}$} \\
\cline { 5 - 9 } & & & $\mathrm{mD} 1$ & $\mathrm{mD} 2$ & $\mathrm{mD} 3$ & $\mathrm{SiD} 1$ & $\mathrm{SiD} 2$ \\
\hline CCRI-25 & 25 & 13.9 & 1.83 & 2.11 & 2.33 & 1.03 & 1.09 \\
CCRI-50(b) & 50 & 22.6 & 2.04 & 2.21 & 2.46 & 1.03 & 1.08 \\
CCRI-100 & 100 & 38.5 & 1.69 & 1.75 & 1.86 & 0.96 & 1.00 \\
CCRI-135 & 135 & 59.4 & 1.37 & 1.40 & 1.43 & 0.95 & 0.96 \\
CCRI-180 & 180 & 77.7 & 1.21 & 1.23 & 1.25 & 0.96 & 0.96 \\
CCRI-250 & 250 & 126.9 & 1.07 & 1.08 & 1.09 & 0.98 & 0.97 \\
\hline \hline
\end{tabular}

